\title{
Shape from planar curves: a linear escape from Flatland
}

Ady Ecker, Kiriakos N. Kutulakos and Allan D. Jepson

University of Toronto

\section{Introduction}

We revisit the problem of recovering $3 \mathrm{D}$ shape from a single photo containing planar curves. Previous work [1,2,3] did not explicitly explore the space of flat solutions and its orthogonat complement

- For intersecting planar curves, we study the space of solutions and derive a stable linear method

For parallel planar curves, we demonstrate special cases where the shape is similarly solved

-Our work unifies relevant literature on shape from contour, single view modeling and structured light

\section{Intersecting curves}

\section{Consider planar curves}

$z_{i}(x, y)=a_{i} x+b_{i} y+d_{i}$

At intersection points

$z_{i}\left(x_{i j}, y_{i j}\right)-z_{j}\left(x_{i j}, y_{i j}\right)=$

$\left(a_{i}-a_{j}\right) x_{i j}+\left(b_{i}-b_{j}\right) y_{i j}+\left(d_{i}-d_{j}\right)=0$

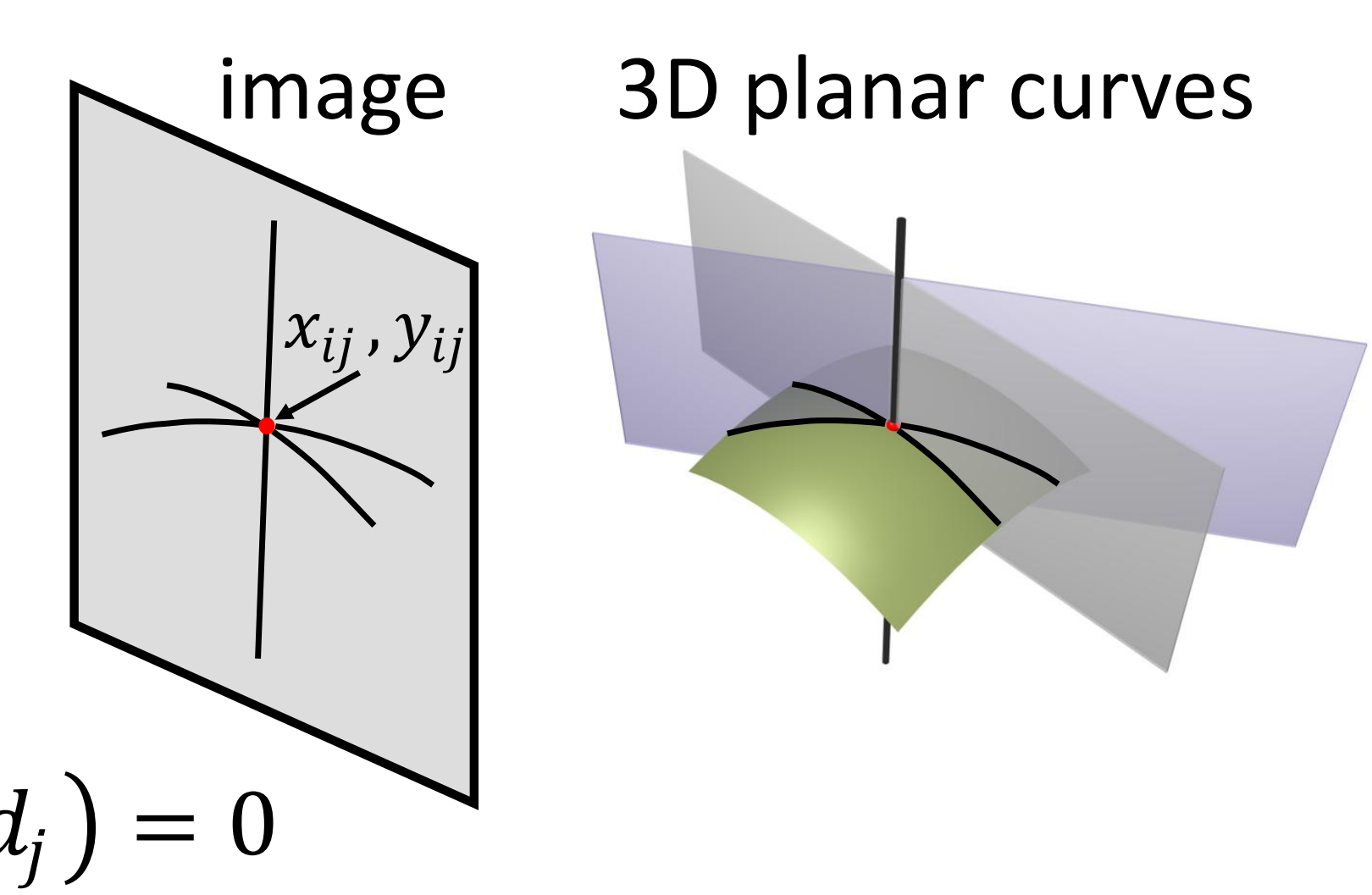

The homogenous linear system in vector form

$\mathbf{A v}=0$

$\mathbf{v}=\left(a_{1}, \ldots, a_{N}, b_{1}, \ldots, b_{N}, d_{1}, \ldots, d_{N}\right)^{T}$

Let $\mathbf{C}$ be a matrix so that $\|\mathbf{C v}\|^{2}$ is the linear regression residue of

fitting a plane to a sample of 3D points on the curves

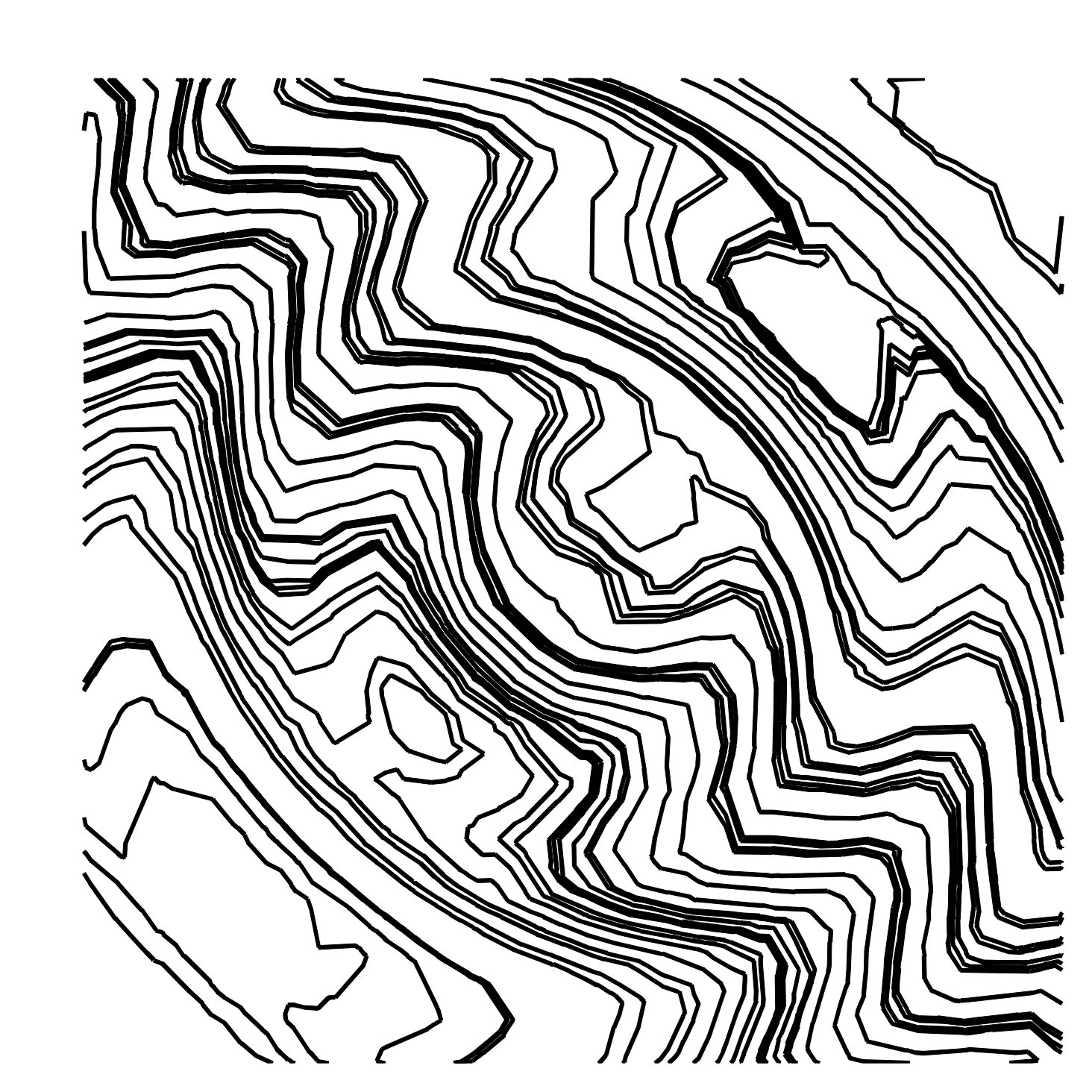

parallel curves

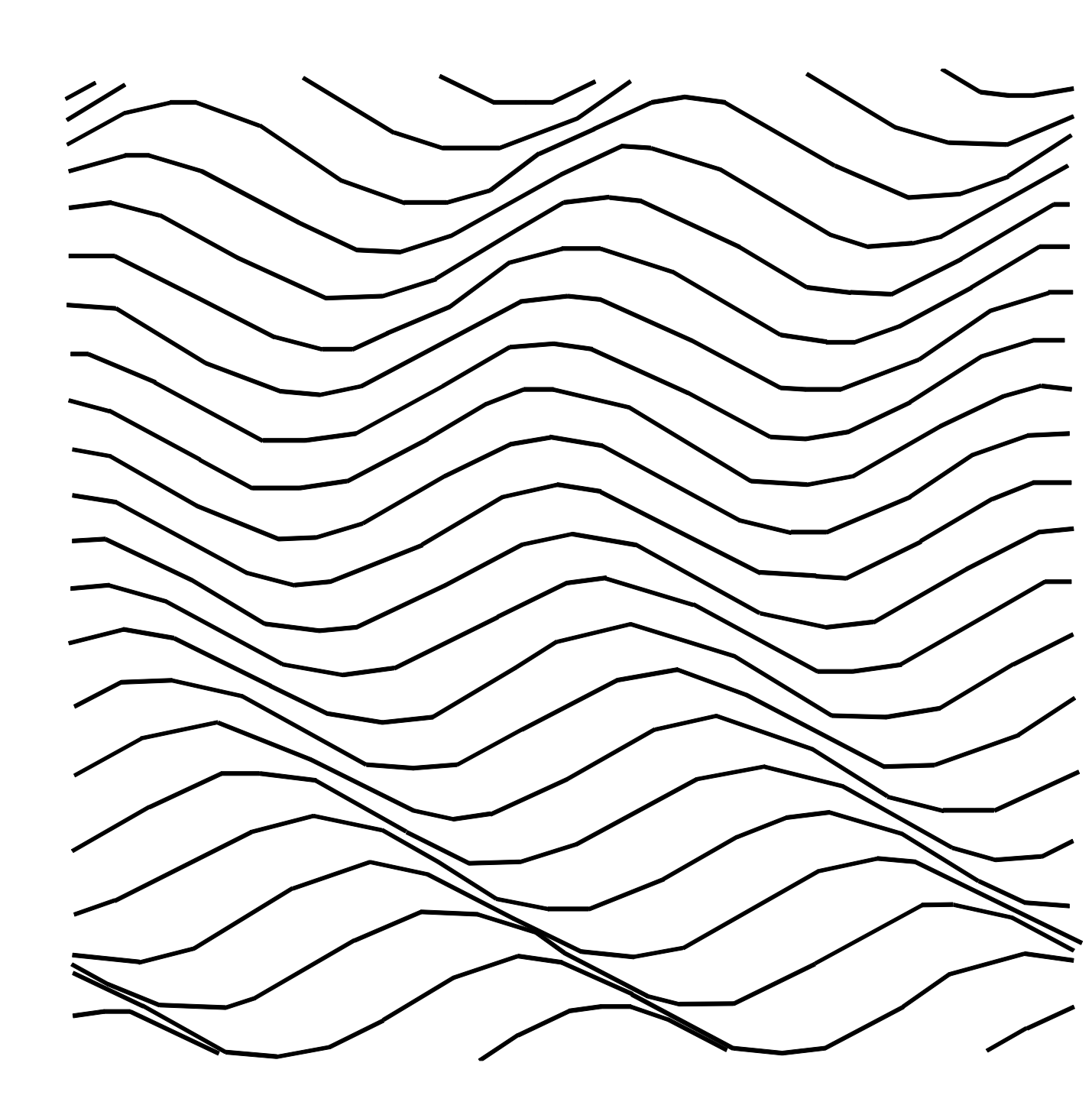

Parallel curves

-Humans can perceive 3D from parallel planar cross sections

Do not assume fixed gaps between the planes. Not sta

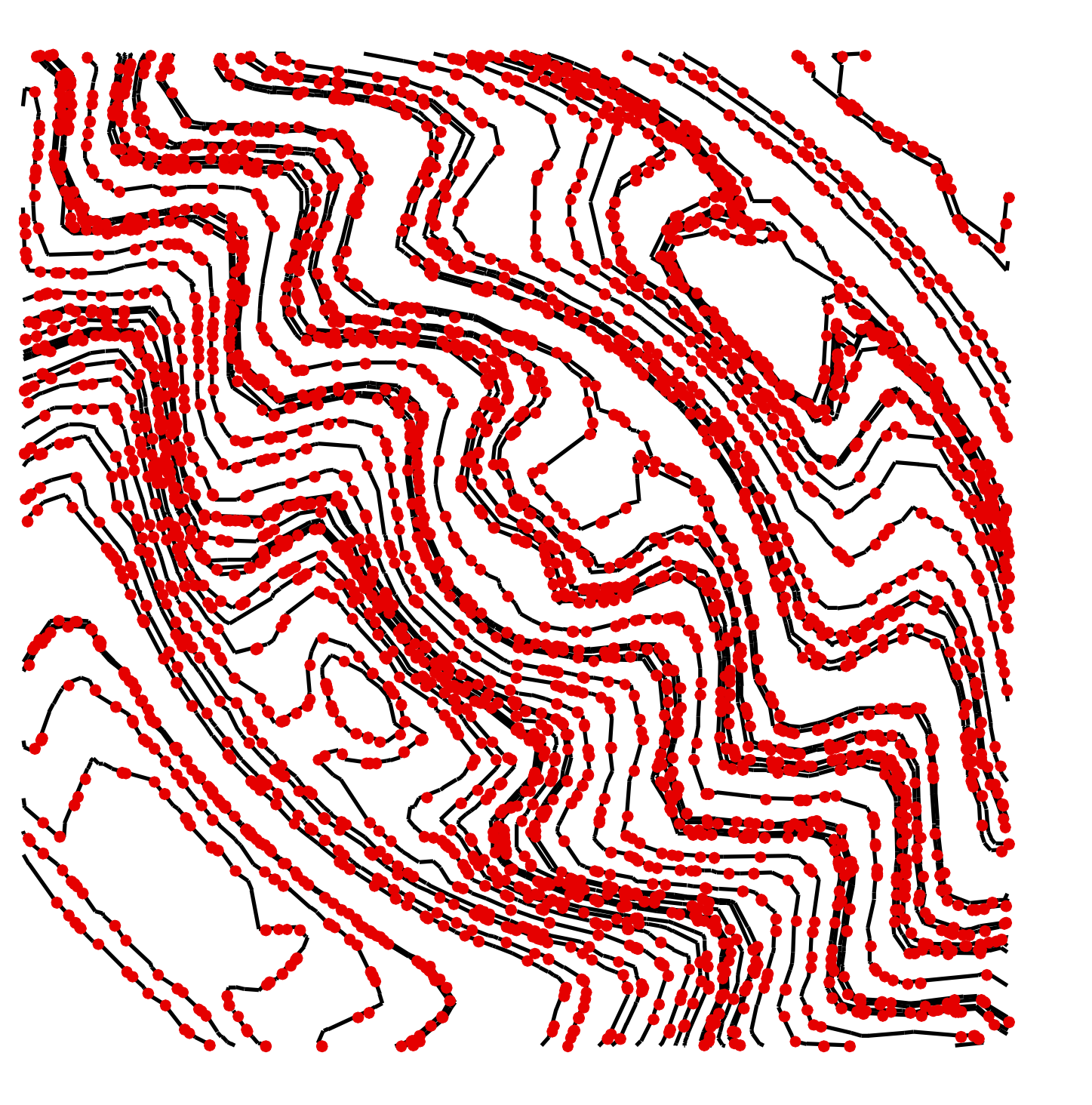

sampled points

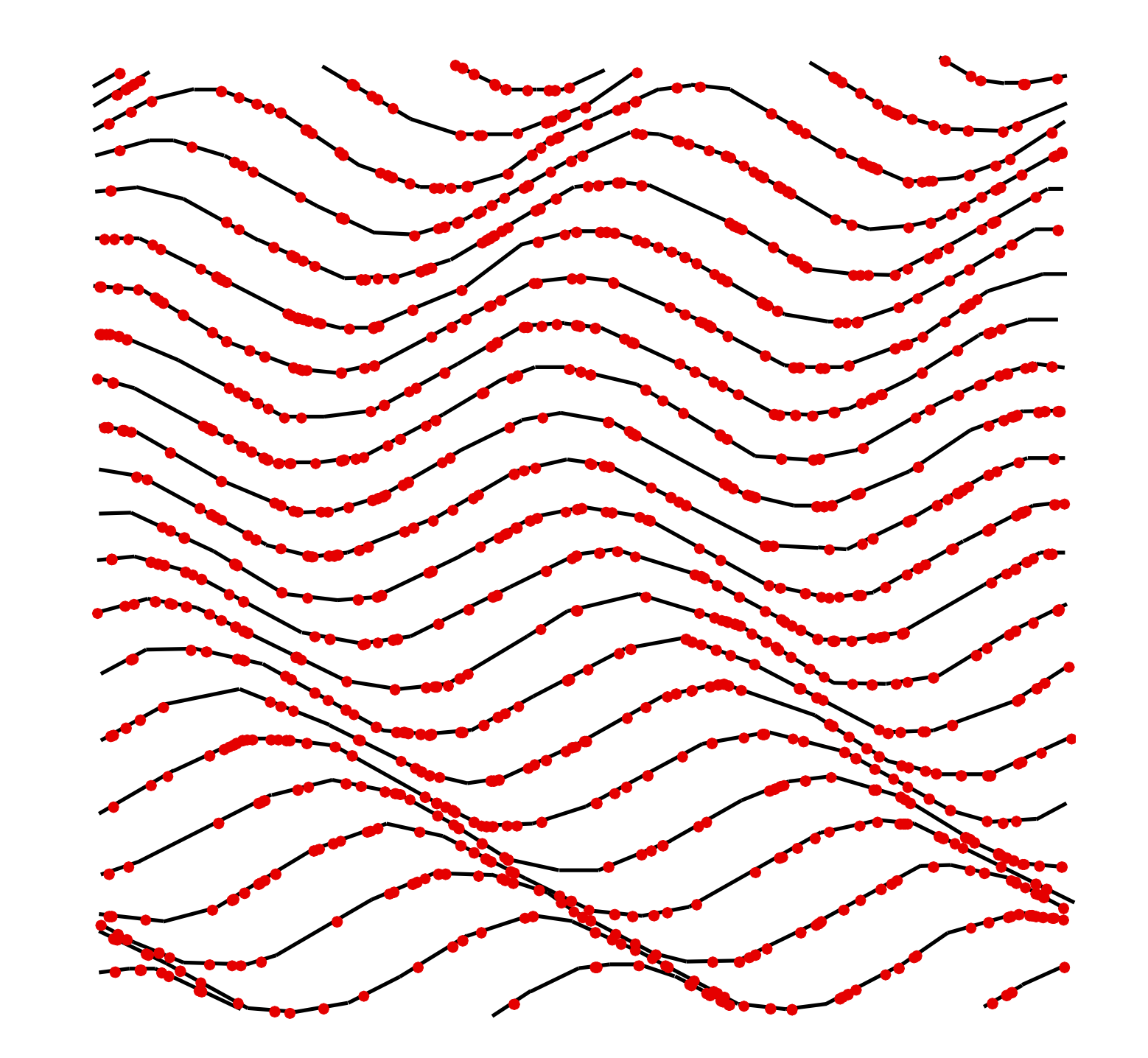

-We demonstrate reconstructions in special cases

\begin{tabular}{|c|c|c|c|}
\hline \multicolumn{4}{|c|}{ Space of solutions $\mathbf{A v}=0$} \\
\hline \multicolumn{2}{|c|}{ Trivial subspace $\mathbf{C v}=0$} & \multicolumn{2}{|c|}{ Non-trivial solutions $\mathbf{C v} \neq 0$} \\
\hline Basic trivial & Systems with lines & \multicolumn{2}{|c|}{ True surface (1D ambiguity) } \\
\hline (GBR ambiguity) & trivial solutions & \multicolumn{2}{|c|}{ Additional non-trivial solutions } \\
\hline$\sqrt{N}$ & & Under-constrained & Over-constrained \\
\hline $\begin{array}{r}\sqrt{N} \\
\mathbf{v}_{3}=\frac{\left(0_{2 N}, N_{N}\right)^{T}}{\sqrt{N}}\end{array}$ & $\begin{array}{l}\text { Straight } \\
\text { curve }\end{array}$ & 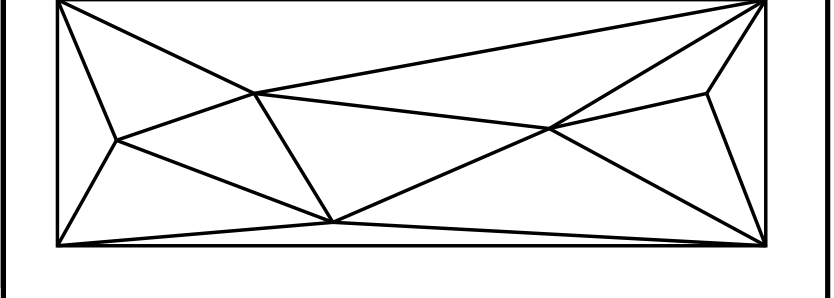 & 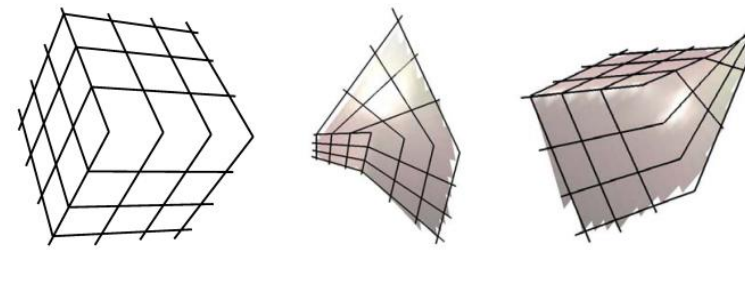 \\
\hline
\end{tabular}

Ambiguity resolution: pick a vector orthogonal to the trivial subspace

- For a large number of random planes, the relative magnitude of the trivial component is likely to be small $\frac{1}{\sqrt{N}}\left\|\left(\sum a_{i}, \sum b_{i}\right)\right\| /\left\|\left(a_{1}, \ldots, a_{N}, b_{1}, \ldots, b_{N}\right)\right\|$

\section{Simple linear method}

$\operatorname{argmin}\|\mathbf{A v}\|$ s.t. $\|\mathbf{v}\|=1, \mathbf{v} \perp \operatorname{Span}\left\{\mathbf{v}_{1}, \mathbf{v}_{2}, \mathbf{v}_{3}\right\}$

- Breaks in presence of straight lines

-May collapse to a nearly-flat solution

Our method

$\underset{\mathbf{v}}{\operatorname{argmin}}\|\mathbf{A v}\|$ s.t. $\|\mathbf{C v}\|=1, \mathbf{v} \perp \operatorname{Null}(\mathbf{C})$

$\mathbf{v}=\mathbf{C}^{+} \mathbf{W}$, where $\mathbf{w}$ is the last right singular vector of $\mathrm{AC}^{+}$

Similar linear formulation for perspective projection

-Singular values are independent of the focal length

(r)
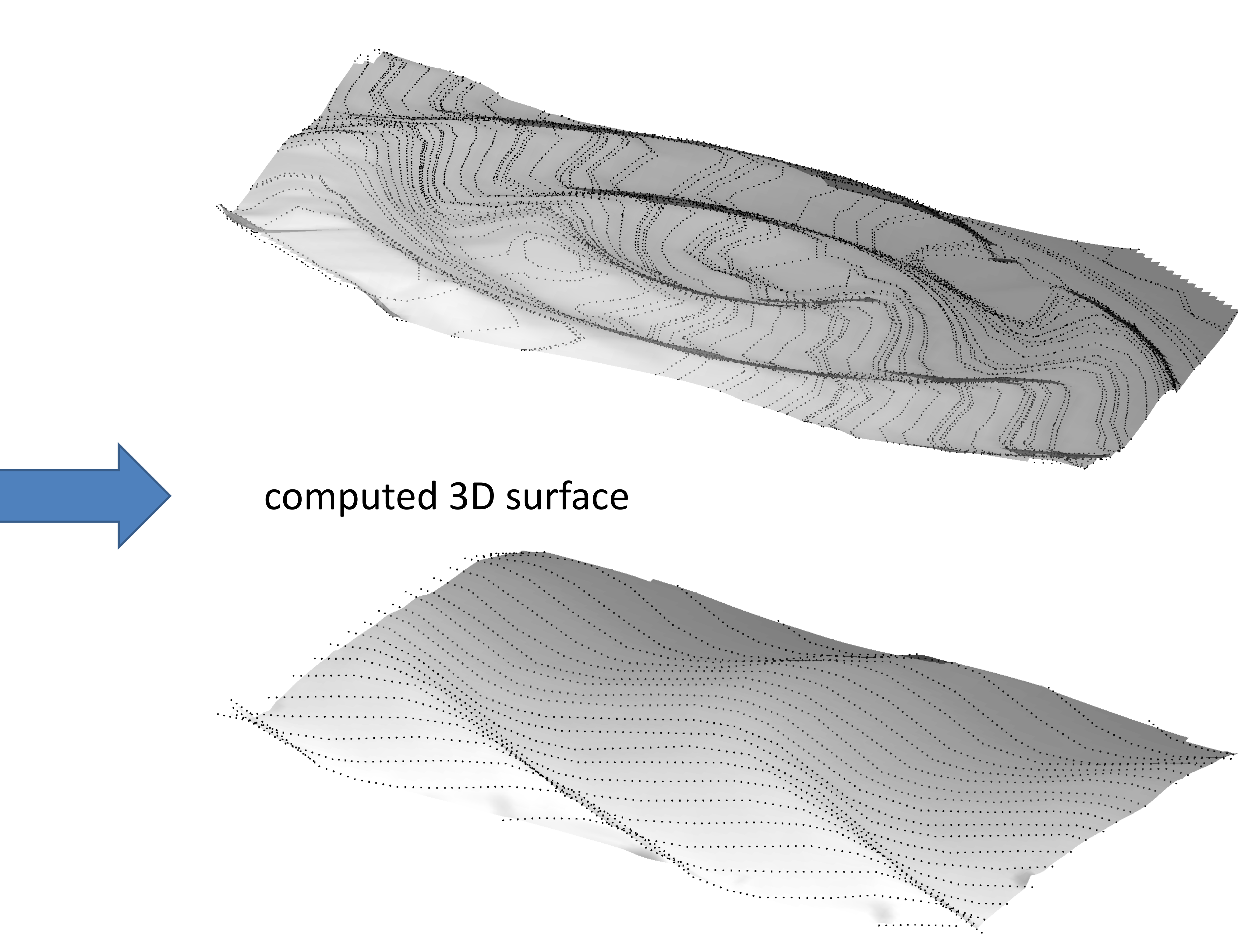

computed 3D surface

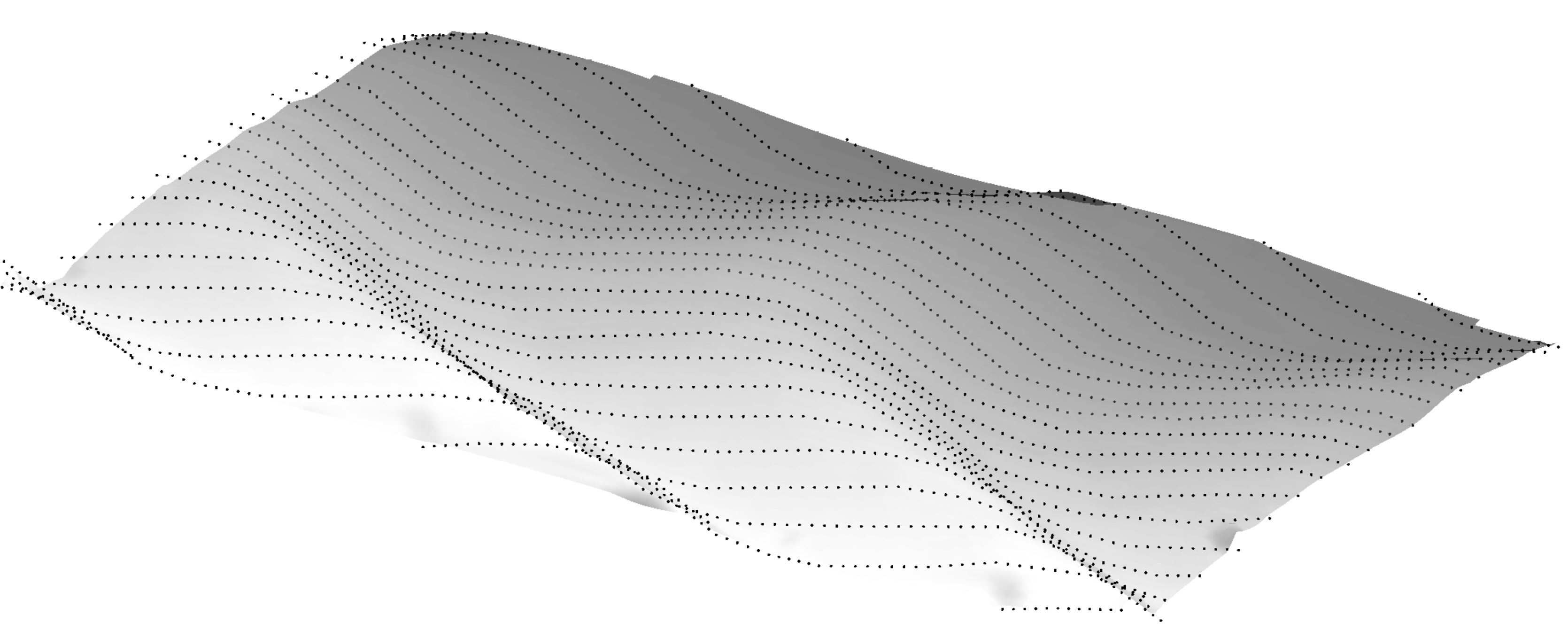

Global planarity

For curves arranged around a principle plane,

the shape might be a singular vector of $\left[\begin{array}{l}\mathbf{R} \\ \mathbf{C}\end{array}\right]$,

$\|\mathbf{R v}\|^{2}=\lambda\left(\operatorname{Var}\left(a_{i}\right)+\operatorname{Var}\left(b_{i}\right)\right)$
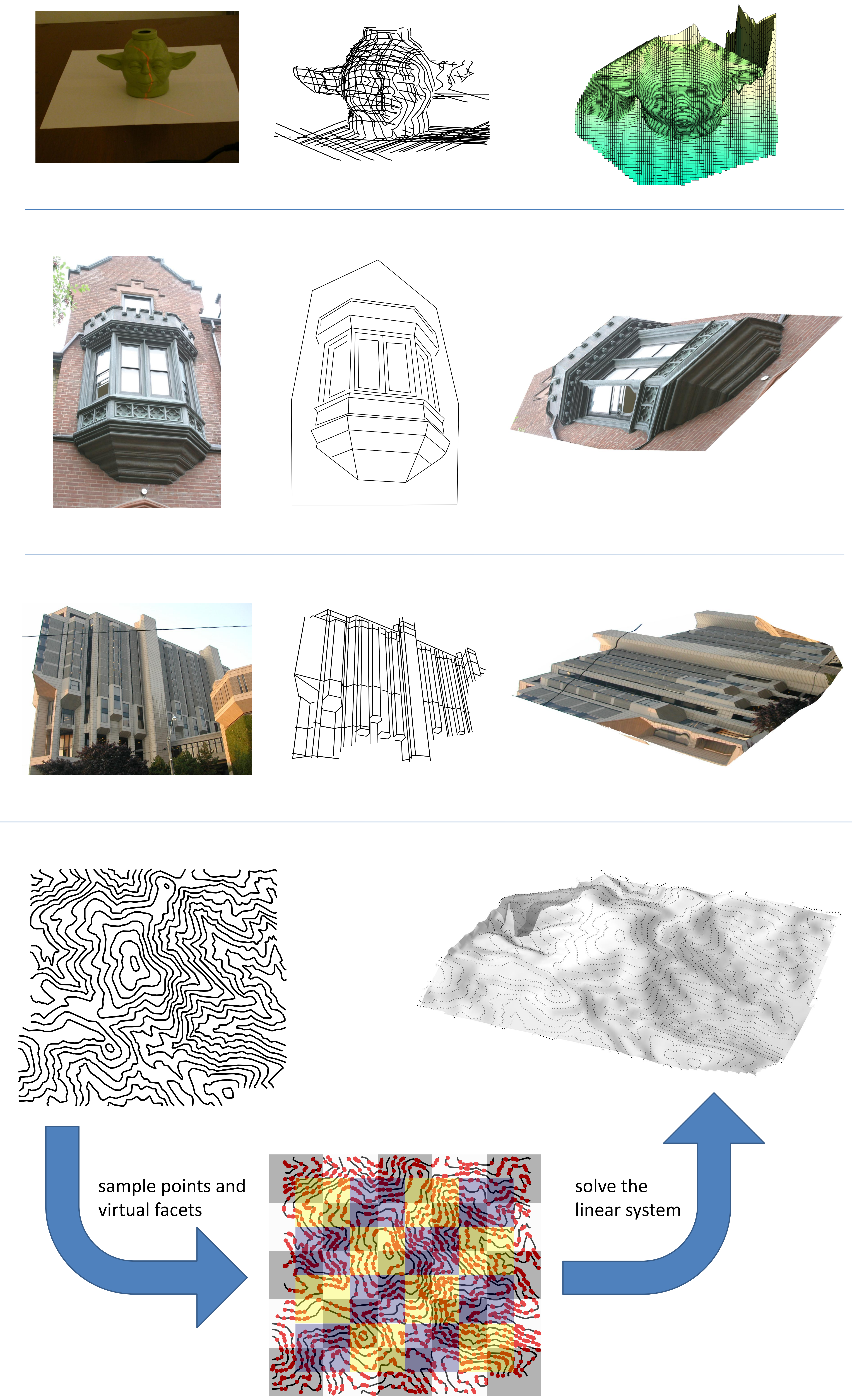

Local planarity Reduce to the intersecting case by assuming virtual

\section{References}

planar facets

[1] K. Sugihara. Machine Interpretation of Line Drawings. MIT press, 198

[2] C. Rothwell and J. Stern. Understanding the shape properties of

trihedral polyhedra. Technical Report 2661, INRIA, 1995

3] J.-Y. Bouguet, M. Weber, and P. Perona. What do planar shadows tell us about scene geometr? In Proc. CVPR'99, pages 514-520, 1999 